\title{
Migrantes europeos en la Costa del Sol: análisis de la audiencia alemana en el consumo de los medios extranjeros en el proceso de integración europea
}

\section{European migrants on the Costa del Sol: an analysis of the consumption of foreign media by the German audience in the process of European integration}

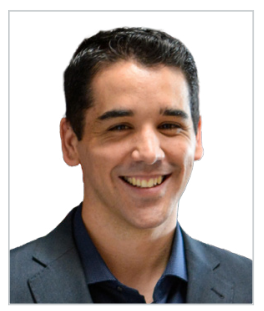

Antonio Cuartero. Investigador Postdoctoral por la Universidad de Málaga (Plan Propio de Investigación) en el Departamento de Periodismo. Doctor en Periodismo con Mención Europea por la Universidad de Málaga. Premio Extraordinario de Doctorado en el programa de Educación y Comunicación Social 2017 por su tesis doctoral. Acreditado como Profesor Ayudante Doctor por la Agencia Nacional de Evaluación de la Calidad y Acreditación (ANECA). Ha disfrutado de un contrato predoctoral de investigación FPU desde 2013 a 2017. Su investigación está centrada en el periodismo narrativo, la crónica y el reportaje. En su etapa postdoctoral ha obtenido diversas contratos y becas de investigación como la Studies Grant form FIAT/IFTA "The International Federation of Television Archives” 2019. Es editor de TSN. Revista de Estudios Internacionales.

Universidad de Málaga, España

cuartero@uma.es

ORCID:0000-0001-9099-8254

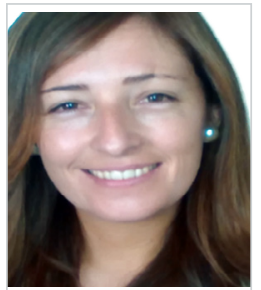

Aida María de Vicente Domínguez. Profesora del Departamento de Periodismo de la Unidad de Málaga. Doctora en comunicación por la Universidad de Granada. Licenciada en Periodismo por la Universidad Carlos III de Madrid y en Humanidades por la Universidad de Navarra. Acreditada como profesora contratada doctora por la Agencia Nacional de Evaluación de la Calidad y Acreditación (ANECA), está especializada en Periodismo Internacional y dirección de comunicación. Pertenece al grupo de investigación HUM 664 de la UMA denominado "Grupo de investigación en comunicación y sociedad de la información.

Universidad de Málaga, España

aidamaria@uma.es

ORCID: 0000-0002-8085-5097

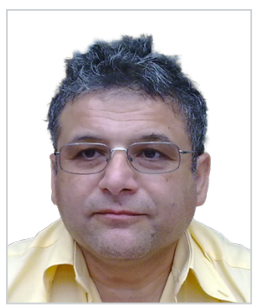

Francisco Báez de Aguilar González. Profesor Titular del Departamento de Filología inglesa, Francesa y Alemana de la Facultad de Filosofía y Letras de la Universidad de Málaga. Doctor en Filosofía por la Universidad de Zúrich. Su actividad investigadora actual se centra en estudios contrastivos interlingüísticos e interdisciplinarios bajo perspectivas sociolingüísticas, pluricentristas, variacionales, lexicográficas u onomásticas.

Universidad de Málaga, España

fbaezdag@uma.es

ORCID: 0000-0002-2536-8130

Cómo citar este artículo:

Cuartero, A.; de Vicente Domínguez, A. M. y Báez de Aguilar González, F. (2020). Migrantes europeos en la Costa del Sol: análisis de la audiencia alemana en el consumo de los medios extranjeros en el proceso de integración europea. Doxa Comunicación, 31, pp. 107-129. https://doi.org/10.31921/doxacom.n31a5 
Recibido: 10/02/2020 - Aceptado: 21/09/2020

\section{Resumen:}

El artículo analiza el papel que desempeñan en el proceso de integración y construcción europea aquellos medios en lengua extranjera publicados en la Costa del Sol para la población alemana residente en esta región. Los objetivos son conocer las características sociodemográficas de esta audiencia activa, identificar su dieta mediática, analizar el papel que desempeñan los medios que componen esta dieta mediática en la integración de la población de residentes alemanes en la sociedad española, especialmente desde el punto de vista del proceso de construcción europea. La encuesta y el grupo de discusión conforman la metodología central de este estudio. La principal conclusión explica que estos medios les ayudan parcialmente en su proceso de integración, si bien, podría aumentar este papel si atendiese a las demandas informativas y los problemas que obstaculizan su integración. Además, los alemanes residentes en la Costa del Sol demuestran un fuerte espíritu europeísta.

Palabras clave:

Residentes alemanes; integración europea; prensa extranjera; residentes extranjeros.
Received: 10/02/2020 - Accepted: 21/09/2020

\section{Abstract:}

This article analyses how the foreign-language media published on the Costa del Sol for the German population living in this region affect their process of European integration and construction. The aims are to determine the socio-demographic characteristics of this active audience, to identify their media diet and to analyse the role played by the media they consume in the integration of the German resident population into Spanish society, especially from the point of view of the process of European construction. Surveys and discussion groups make up the core methodology of this study. The main conclusion is that these media help them to an extended in their process of covered, although this role could be further expanded if the media met the information demands and the problems that hinder the residents' integration. Furthermore, the Germans living on the Costa del Sol display a strong pro-European spirit.

\section{Keywords:}

German residents; European integration; media of diaspora; foreign residents.

\section{Introducción}

La Costa del Sol es, desde hace décadas, uno de los destinos preferentes de la población europea para fijar una nueva residencia, lo cual ha generado en este espacio geográfico un curioso fenómeno mediático: el surgimiento de medios extranjeros destinados a estas poblaciones en su idioma. Este hecho ha provocado un inusual escenario. Y es que, si cualquier colectivo de migrantes tiene dos ecosistemas mediáticos con los que convive normalmente, los medios de su país de origen y los medios del país de destino, en el caso de la Costa del Sol (y otras zonas de España y Portugal) también conviven con un tercer ecosistema: los medios producidos, exclusivamente, para estas poblaciones en su propia lengua, y en la mayoría de los casos gestionados por europeos que llevan ya desde hace décadas viviendo en esta zona.

En este contexto migratorio, poblacional y mediático europeo nuestra investigación se centra en la población de alemanes que reside en la Costa del Sol${ }^{1}$. Representan estos la segunda nacionalidad europea en volumen de población y su número asciende a $9.456^{2}$ residentes según los datos proporcionados por el Instituto Nacional de Estadística en 2016, si bien el número de alemanes registrados en los ayuntamientos (como se mostrará a continuación) es muy inferior al de aquellos que realmente residen al menos una parte importante del año en la Costa del Sol. A falta de censos específicos exhaustivos, el INE representa la fuente más fiable también para analizar este fenómeno migratorio.

1 Entendemos el espacio geográfico Costa del Sol como la región litoral del sur de la provincia de Málaga incluyendo en ellos todos aquellos municipios circundantes a la costa con mayoría de residentes extranjeros.

2 Véase: https://www.ine.es/dyngs/INEbase/es/operacion.htm?c=Estadistica_C\&cid=1254736177012\&menu=resultados\&idp=1254734710990 
El presente estudio resulta significativo por su perspectiva innovadora desde la que pretende determinar la relevancia de los medios extranjeros para alemanes en el proceso de integración y construcción de Europa. Se centra en el análisis del consumo mediático de la población alemana residente en la Costa del Sol, que no sólo representa el segundo grupo poblacional europeo en la región, sino también el segundo en cuanto al número de medios en su propio idioma, según el censo que se ha realizado en este proyecto.

Investigación con la que se pretende aprehender y comprender mejor el proceso general para alcanzar la meta de una Europa unida en su pluralidad; y esto en una fase especialmente crítica del proyecto europeo debido al Brexit y la ola de euroescepticismo de numerosos partidos europeos que no dejan de nutrir los miedos surgidos entre los pueblos de Europa ante el proceso de integración. Resulta, por tanto, más necesario que nunca analizar por un lado cómo ven los europeos deslocalizados todo este proceso y el papel que desempeñan o han de desempeñar los medios españoles en lengua alemana y los medios alemanes distribuidos en España, y por otro, ver como los medios en alemán difundidos en la Costa del Sol, fomentan o determinan la comprensión, cohesión, integración y, en el fondo, ayudan a alcanzar la meta del proyecto europeo.

Esta investigación, junto con las restantes del proyecto del que forma parte, ayudará igualmente a obtener una radiografía sobre las poblaciones de migrantes europeos como audiencias activas, o al menos de una parte importante de ellas.

\section{Estado de la cuestión}

Las primeras investigaciones en torno a los medios extranjeros y la información publicada en otros idiomas en la Costa del Sol y España surgen con la llegada de los primeros migrantes y turistas en masa en los años 70 (García Galindo 1998), aunque como constatan García Galindo y López Romero (2013) y López Romero y Serrano Porras (2016) ya desde inicios del siglo XX, se viene observando la publicación de informaciones en otros idiomas en la prensa de la Costa del Sol. Esta larga presencia de informaciones en otros idiomas y la existencia de medios extranjeros en la Costa del Sol, las Islas Canarias, la costa valenciana y en otras zonas de España no se han traducido, sin embargo, en una amplía investigación en torno a este fenómeno. En general, la producción científica sobre los medios extranjeros no es muy abundante y, entre esta, el grupo que más atención ha recibido es el de los británicos, seguramente por representar la principal población de extranjeros asentados en la Costa del Sol (Karen O’Reilly 2009; Betty 1997; Betty y Duran 2008; Rodríguez, Fernández-Mayoralas, y Rojo 2004; Villaverde y Hierro 2013). Otros autores han abordado a los extranjeros desde un punto de vista más general (García Galindo y López Romero 2018; Oliveau et al. 2019). Nuestro estudio pretende ahora profundizar también en la situación de los ciudadanos alemanes, la segunda población europea en número de residentes en la Costa del Sol.

Las investigaciones precedentes sobre los residentes comunitarios alemanes en España son escasas y ninguna aborda el fenómeno en la Costa del Sol. Dorn Padilla (2012) investiga el caso de los turistas alemanes en las Islas Canarias, pero no el de los residentes alemanes, como es nuestro caso. Su estudio resulta, no obstante, de gran interés porque analiza la prensa destinada a esta comunidad en las Islas Canarias y evidencia que también se trata de la segunda comunidad europea con más presencia en ellas. Además, señala la repercusión que tiene el turismo alemán en el desarrollo de actividades periodísticas en esta lengua y analiza de manera especial las funciones del periodismo de servicios que desempeñan estos medios a través de sendas vías, la de la cohesionar a esta población y la de facilitar su estancia. 
Penalva y Brückner (2008) analizan el periódico Costa Blanca Nachrichten ${ }^{3}$ versión alicantina del tema tratado en esta investigación y concluyen que no se trata de un medio que transmite las demandas sociales, y que, en términos de interculturalidad, tampoco es percibido como un medio híbrido, sino que funciona más bien como un medio intercultural entre la población alemana. Brückner (2008) corrobora estos resultados en otra investigación sobre el mismo periódico.

El resto de las investigaciones sobre los medios extranjeros se han centrado en otras nacionalidades y áreas geográficas, por ejemplo, en las Islas Canarias (Acirón Royo 1997; Martín Hernández 1990; González Cruz, 1991), en las Islas Baleares (Marimon y Vicens 2013) o en Madrid (Retis 2008). En esta última Comunidad, se han realizado igualmente investigaciones sobre residentes latinoamericanos (Gómez y Santín, 2009; Mas Giral 2017).

Otros investigadores abordan la problemática de este tipo de prensa son el de la gratuidad de los medios (Santos Díez y Pérez Dasilva 2012; Rosell 2008; González Cortes 2009; Santos Díez 2008), el de los estudios de traducción (Taillefer de Haya 2005), y, por supuesto, el del turismo, desde cuyo ámbito se ha analizado con profusión otras nacionalidades (Fernández y Mendoza 2007; Ocaña y Larrubia 2012; Mazón 2018; Delgado Peña y Sortino Barrinuevo 2018; Millares 2018; Lakssfoss 2018; Woube 2017). También la televisión le ha dedicado cierta atención a la temática en un estudio de audiencias centrado en los jóvenes británicos en la Costa del Sol (Sáiz Díaz 2016).

El presente estudio pretende aportar nuevos datos a este campo de estudio cuya conceptualización no está aún del todo consensuada. En la bibliografía consultada se detectan diversas terminologías: prensa étnica, prensa en la diáspora, prensa inmigrante, prensa extranjera, prensa para minorías o prensa deslocalizada (Blau 1998; Lacroix 1998; Gómez Mompart 2008). En esta investigación se opta por el termino más frecuente, «prensa extranjera», para estudiar el caso de la segunda de las poblaciones europeas más numerosas en la Costa del Sol, y también en el resto de España, la alemana, pero que a pesar de ello, menos atención académica ha recibido.

\section{Objetivos y Metodología}

La hipótesis de partida de esta investigación considera que los residentes alemanes en la Costa del Sol están a favor del proyecto europeo y que los medios destinados a este colectivo les ayudan en su proceso de integración. Pero estos presentan deficiencias pues no atienden a todas las demandas informativas que precisan como residentes extranjeros en esta área geográfica.

3 Este periódico tiene otras dos versiones, una destinada a la Costa del sol con el nombre de Costa del Sol Nachrichten y otra destinada a la región de Murcia con el nombre de Costa Cálida Nachrichten. 
Los objetivos fijados en esta investigación son tres:

1. Conocer las características sociodemográficas de esta audiencia activa -los alemanes residentes- en la Costa del Sol.

2. Identificar la dieta mediática de la población alemana residente en la Costa del Sol.

3. Analizar el papel que desempeñan los medios que componen esta dieta mediática en la integración de la población de residentes alemanes en la sociedad española, especialmente desde el punto de vista del proceso de construcción europea.

La metodología utilizada aquí es la desarrollada para el proyecto I+D+i "Medios de Comunicación y construcción europea: estudio sociocomunicativo de los residentes comunitarios en el sur de España y de Portugal (CSO2015-65837-R)" en su totalidad. Este proyecto I+D+i tiene una vigencia de enero 2016 a diciembre 2019 y está dirigido por el catedrático de Periodismo Juan Antonio García Galindo. Centra su investigación en aquellas comunidades de migrantes europeos con mayor representación mediática en la Costa del Sol y el Algarve: alemanes, daneses, fineses, franceses, holandeses, británicos, suecos y noruegos. Finalmente, se estimó oportuno y necesario incluir a la población rusa por su proporción de residentes y su alta representación mediática, y no hacerlo en el caso de otros comunitarios como italianos o ucranianos porque carecen de medios publicados en su idioma.

La metodología está estructurada en dos bloques metodológicos con un total de cinco fases. Evidentemente, a lo largo de todo el proyecto los contenidos de estas fases se han ido actualizando con nuevas informaciones o aspectos surgidos y en algún momento unas fases se han desarrollado en paralelo a otras. El primer bloque engloba tres fases.

Fase 1: Elaboración de una base de datos en línea que permita volcar los resultados obtenidos de la búsqueda bibliográfica sobre los medios extranjeros. La búsqueda de estos medios se realizó en bases de datos nacionales e internacionales (Dialnet, Rebiun, Teseo, B-on, Google Academic, y Scopus), y las palabras claves o ecuaciones de búsqueda usadas fueron: "prensa extranjera", "medios extranjeros", "medios para inmigrantes", "prensa para inmigrantes", "medios comunitarios", "medios deslocalizados", "prensa deslocalizada" "prensa extranjera Costa del Sol” "prensa y turismo Costa del Sol", añadiendo a continuación de estos términos la nacionalidad analizada, que en el caso objeto de nuestro estudio es la alemana.

De cada documento se optó por insertar el tipo de texto (libro, artículo, capítulo...), el título, el nombre del autor, el año de la publicación y su editor, el enlace de internet (si lo tuviera), las disciplinas (acotadas a cinco palabras como máximo), palabras clave, la referencia bibliográfica completa en formato APA, la relevancia para el proyecto y observaciones. En total se localizan 203 aportaciones generales y cinco de ellas analizan la población alemana, pero sólo tres se centran en los medios destinados a esta población. 
Imagen 1. Base de datos bibliográfica

\begin{tabular}{|c|c|c|}
\hline Libro, artículo, tesis... & Título & Autor \\
\hline \multicolumn{2}{|c|}{ Prensa (Recortes de pren Torrox, 'la pequeña Alemania' de Málaga } & José Carlos Pozo \\
\hline \multicolumn{2}{|c|}{ Artículos de revistas cient Fixed laws, fluid lives: the citizenship status of post-retirement migrants in the European Union } & Ackers, L. and Dwyer, P. \\
\hline Libros & The British on the Costa del Sol & Karen O'Reilly \\
\hline \multicolumn{2}{|c|}{ Informes/Estadísticas/Anı Observatorio turístico Andalucía-Algarve. Demanda turística } & CEAL, FOE, CEA, UE, JL \\
\hline Libros & A Comunicación Social Transfronteiriza & Alberto Pena Rodríguez \\
\hline Capítulos de libro & Historia del Periodismo Portugués & Alberto Pena Rodríguez \\
\hline \multicolumn{2}{|c|}{ Tesis doctorales y otros tr Portugal en América. La prensa portuguesa en los Estados Unidos de América: inmigración periodismo y propaganda (1877-201: } & Alberto Pena Rodríguez \\
\hline Libros & Emigraciion e exilio nos Estados Unidos de América. Experiencias de Galicia e Azores & Alberto Pena, Mário Mesc \\
\hline Artículos de revistas cient & I El papel de los destinos turísticos en la transformación sociodemográfica del litoral mediterráneo español & Francesc González Reve \\
\hline Artículos de revistas cien & 1 La actividad turistica en el territorio andaluz. Establecimiento de indicadores, distribución y evolución de los mismos & Alfonso Fernández Tabalє \\
\hline Artículos de revistas cien & Residentes extranjeros y crecimiento demográfico en el territorio andaluz & María del Carmen Ocaña \\
\hline \multicolumn{2}{|c|}{ Artículos de revistas cient La consolidación de las publicaciones para extranjeros en España } & María Eugenia González \\
\hline Libros & Recursos, potencialidades y modelos turísticos en el Baixo Alentejo, Algarve y provincia de Huelva & José Manuel Jurado Almc \\
\hline \multicolumn{2}{|c|}{ Artículos de revistas cient Extranjeros en Andalucíadiversificación de las corrientes inmigratorias y sus patrones de distribución sobre el espacio andaluz } & Remedios Larrubia Varga \\
\hline Libros & Practising the Good Life: Lifestyle Migration in Practices & Kate Torkington, Inês Dav \\
\hline Capítulos de libro & Glocalización comunicativa y prensa local extranjera en España: La visión de los residentes alemanes de la Costa Blanca. & Brückner, G. \\
\hline \multicolumn{2}{|c|}{ Artículos de revistas cient Comunicación intercultural. Un estudio de caso sobre la prensa local extranjera en España. } & Penalva, C. y Brückner, G \\
\hline Libros & La integración de los extranjeros: un análisis transversal desde Andalucía & Sánchez Lorenzo, S. (ed. \\
\hline Libros & Voces de la inmigración, medios latinos en Madrid. & Gómez-Escalonilla, G. (cc \\
\hline
\end{tabular}

Fuente: elaboración propia

Fase 2: Elaboración de un censo de medios para obtener una radiografía de la situación actual respecto a los medios de comunicación destinados a los extranjeros en la Costa del Sol. La elaboración de este censo, que en el presente estudio se centra en los medios alemanes, parte de la estructura diseñada por López Romero (2009) en su tesis doctoral sobre este campo de estudio que considera los siguientes apartados de análisis: zona geográfica, nombre del medio, tipología del medio (periódico, revista, canal de televisión...), dial (en su caso), año de inicio, año de finalización, periodicidad, idioma, (señalando si es más de uno), sede completa, existencias, imprenta, director y redactores, titularidad, geografía de la difusión, orientación y naturaleza del medio, datos de contacto y observaciones.

El censo realizado de enero de 2016 a mayo de 2018 contiene los medios existentes en prensa, prensa digital y radio en la Costa del Sol. Si bien, cabe destacar que en este censo figuran varios medios que han sido cerrados en el transcurso del período estudiado. En total se han localizado 130 medios, 112 en la Costa del Sol y 18 en Algarve. Los métodos desarrollados para completar esta base de datos fueron tres: una búsqueda documental y bibliográfica a través de la red para detectar nuevas publicaciones; entrevistas a periodistas y responsables de medios extranjeros conocidos para que nos comunicaran la existencia de otros medios de su entorno; y una búsqueda de campo visitando lugares turísticos y de gran afluencia de turistas, donde muchas de estas publicaciones se dejan de forma gratuita. 
El total de medios detectados destinados a la población alemana en la Costa del Sol son los siguientes:

- SUR Deutsche Ausgabe

- Costa del Sol Nachrichten

- Spanien Aktuell

- Das Aktuelle Spanienmagazin

- SAZ-Aktuell

- Der Marktplatz

Aunque hay otros siete medios que ofrecen algún apartado o sección con información en alemán:

- Andalucía Golf Guide

- andaluz.tv

- Economía Hispano Alemanal Deustsch-Spanische Wirtschaft

- XtraFM

- A Spanish Life

- Finest Magazine Marbella

- Ronda Magazine

Imagen 2. Captura de pantalla parcial de la muestra del censo de medios realizados

\begin{tabular}{|c|c|c|c|c|c|}
\hline C & D & $E$ & $\mathrm{~F}$ & G & $\mathrm{H}$ \\
\hline Nombre completo del medio & Tipologia del medio & Dial & Año de inicio & Año de finalización & Periodicidad \\
\hline Andalucía Golf Guide & Guía & & 2005 & Acaba dentro del periodo & Sin periodicidad regular \\
\hline ミSUR deutsche Ausgabe & Periódico & & 2004 & sigue & Semanal \\
\hline ミCosta del Sol Nachrichten & Periódico & & 1996 & sigue & Semanal \\
\hline ¿Spanien aktuell & Revista & & Desconocido & sigue & Mensual \\
\hline :Das aktuelle Spanienmagazin & Revista & & Desconocido & sigue & Mensual \\
\hline andaluz.tv & Prensa digital & & 2009 & sigue & Sin periodicidad regular \\
\hline Economía Hispano alemana / Deutsch-Spanische Wir & Prensa digital & & 1953 & sigue & Bimestral \\
\hline ESAZ-aktuell & Prensa digital & & Desconocido & sigue & Diaria \\
\hline XtraFM & Radio digital & & 2006 & sigue & Diaria \\
\hline Der Marktplatz & Revista & & 2013 & sigue & Bimestral \\
\hline A Spanish Life - Marbella & Prensa digital & & 2005 & sigue & Sin periodicidad regular \\
\hline Finest Magazine Marbella & Guía & & 1973 & sigue & Bimestral \\
\hline
\end{tabular}

Fuente: elaboración propia

Fase 3: Elaboración de una base de datos que permita volcar contactos de relevancia para el proyecto. Los contactos localizados en los principales puntos de referencia de la población extranjera, en nuestro estudio la alemana en la Costa del Sol, nos deberían ayudar a distribuir las encuestas y a reclutar participantes dispuestos a formar parte de los grupos de discusión.

La ficha de esta base de datos incluye diez apartados: Asociaciones, Federaciones y clubes; Departamento(s) de extranjeros; Consulado(s); Centros educativos extranjeros; Universidades; Centros religiosos; Aeropuertos; Estaciones de ferrocarril; Puertos; Personas; Otros. Para cada institución u organización listada se han introducido los datos del contacto y su utilidad para el proyecto. En total, de los 78 contactos obtenidos para todas las nacionalidades del proyecto general se hace la siguiente selección de colaboradores para el estudio sobre los residentes alemanes: 
- Marion Fischer, organizadora de encuentros de alemanes en el Restaurante Lizzarran en Arroyo de la Miel (Málaga) para distribuir las encuestas.

- El grupo de encuentro hispano-alemán organizado por Intercambio Deutsch-Español en Arroyo de la Miel (Málaga) en el Sprizze One.

- Justo Sánchez Arévalo, administrador de Fincas en Torrox Costa, que atiende numerosas comunidades de propietarios de edificios y urbanizaciones de la Axarquía, muchas de ellas con un gran número de residentes alemanes.

Esta selección tiene igualmente en cuenta la ubicación, costa oriental o costa occidental, en tanto ambas zonas de la Costa del Sol, separadas por la capital misma, muestran una distribución sociodemográfica muy distinta en cuanto a la distribución y composición de la población autóctona, así como de las comunidades de residentes y turistas extranjeros y españoles que explicaremos más abajo.

El segundo bloque metodológico incluye las dos últimas fases. Fase 4: Establecimiento del número mínimo de encuestados necesarios para cada idioma y elaboración y distribución del cuestionario para recopilar datos sobre la dieta mediática, el nivel de integración, la situación personal, y la opinión particular de los encuestados sobre el proceso de construcción europea.

El número de potenciales residentes por nacionalidad en la Costa del Sol se determina a partir de la información oficial ofrecida por el INE respecto a la población extranjera europea residente en Málaga. La cifra de 89.917 $7^{4}$ residentes extranjeros que da esta institución (para el total de nacionalidades extranjeras seleccionadas) en 2016 resulta bastante inferior al número estimado generalmente, pero son los únicos datos oficiales de los que se dispone.

Las características que ha de cumplir la muestra de residentes extranjeros vienen determinadas por un diseño de muestreo simple con cuotas proporcionales por sexo y edad, ajustado al perfil del extranjero europeo en la Costa del Sol, así como al universo poblacional sobre el que se deberán inferir los resultados de la encuesta. El error muestral aplicado es del $+3,45 \%$ y el nivel de confianza del $95,5 \%$, por lo que la muestra requiere 800 encuestas. A partir de estas se calcula proporcionalmente el número de encuestas necesarias por nacionalidad. Resulta así la cantidad de 84 encuestados necesarios para el estudio sobre la comunidad de residentes alemanes. La distribución proporcional de todas las encuestas se refleja en la siguiente tabla:

4 Datos obtenidos del padrón continuo del INE:

https://www.ine.es/dyngs/INEbase/es/operacion.htm?c=Estadistica_C\&cid=1254736177012\&menu=resultados\&idp=1254734710990

114 | n³1, pp. 107-129 | doxa.comunicación 
Imagen 3. Distribución proporcional de las encuestas necesarias por nacionalidades

\begin{tabular}{|l|c|c|c|}
\hline Nacionalidad & No Residentes Extranjeros & Porcentajes & No Encuestas \\
\hline Alemania & 9456 & 10,5 & 84 \\
\hline Dinamarca & 3732 & 4,2 & 33 \\
\hline Finlandia & 5413 & 6,0 & 48 \\
\hline Francia & 5852 & 6,5 & 52 \\
\hline Holanda & 6075 & 6,8 & 54 \\
\hline Reino Unido & 46451 & 51,7 & 413 \\
\hline Suecia & 4606 & 5,1 & 41 \\
\hline Noruega & 2156 & 2,4 & 19 \\
\hline Rusia & 6176 & 6,9 & 55 \\
\hline Total & 89917 & 100,0 & 800 \\
\hline
\end{tabular}

Fuente: elaboración propia a partir de datos del INE

Dado que los encuestados debían cumplir una serie de requisitos en cuanto a la duración de su residencia en la Costa del Sol y siempre hay encuestas que se han de anular por no cumplir los requisitos o estar incompletas se distribuyó y recopiló un número bastante superior de cuestionarios. Fue posible así mantener un buen equilibrio entre las diferentes variables complementarias como, por ejemplo, hombre-mujer, edad, o duración y lugar de la residencia.

El cuestionario redactado y consensuado inicialmente en español para el proyecto general, se tradujo a la lengua correspondiente de las otras nacionalidades investigadas por traductores profesionales, a los que previamente se les había explicado el sentido y el objetivo de cada pregunta de modo que la traducción fuese lo más exacta posible. Antes de comenzar la difusión de los cuestionarios se llevó a cabo un prestest con las versiones online de los idiomas inglés y alemán, desde el 30 de noviembre de 2017 hasta el 25 de diciembre de 2017, para corregir posibles errores.

El cuestionario en alemán contiene, por tanto, al igual que en las otras lenguas las mismas 41 preguntas de tres tipos -cerradas, semi-abiertas y abiertas-, agrupadas en 5 bloques temáticos: mudarse a España (6 preguntas); relaciones sociales (5 preguntas); integración (11 preguntas); medios de comunicación de su país de origen (3 preguntas), y medios de comunicación producidos en la Costa del Sol (16). Los cuestionarios se difundieron en papel y online entre noviembre de 2017 y marzo de 2018.

Y la última, la Fase 5, consiste en la realización de un grupo de discusión para profundizar en aquellas cuestiones que las encuestas individuales no alcanzaban a explicar, y obtener en la discusión nuevas informaciones o matizaciones. El grupo de discusión se realizó el 9 de noviembre de 2018 en las instalaciones de la Facultad de Ciencias de la Comunicación de Málaga estando presentes los 7 participantes (4 hombres y 3 mujeres) un moderador, un observador y un intérprete. La parte oficial de la sesión, grabada con una cámara de video, duró una hora y media. Al principio de cada bloque temático, el moderador invitaba a los participantes a intervenir sucesivamente y a continuación se permitía el desarrollo de una discusión libre, que sólo era interrumpida para que el intérprete pudiera ir traduciendo los comentarios de todos los participantes. La información fue posteriormente transcrita y traducida al español, al igual que 
se ha hecho con los resultados de todas las encuestas escritas y de los otros 7 grupos de discusión por nacionalidades del proyecto general.

\section{Resultados}

\subsection{Características de la estructura sociodemográfica de la audiencia alemana en la Costa del Sol}

Las poblaciones de extranjeros residentes en la Costa del Sol, tradicionalmente, se han repartido en ciertas zonas geográficas, y en el caso de los alemanes no es una excepción. Los datos obtenidos de las encuestas desvelan que están asentados principalmente en Vélez Málaga (20\%), Torrox ${ }^{5}(12 \%)$ y Marbella (9,5\%). Si bien, para interpretar estos datos se subraya que cuando nos referimos a Vélez Málaga hay que incluir a sus pedanías limítrofes como Torre del Mar o Benajarafe, y cuando se hace referencia a Torrox hay que incluir su pedanía de Torrox Costa.

Estos residentes se ubican principalmente aquí, probablemente, porque en Algarrobo Costa y en Torre del Mar, a finales de los 60 y principio de los 70, empezaron a construirse bloques de apartamentos para atraer a la clase trabajadora y media alemana. Los constructores eran alemanes cómo la empresa Bauoffman; o españoles con relaciones personales con alemanas, como la promotora Toto, cuyo propietario estaba casado con una alemana. Y destacable es también que en otros muchos casos, alemanes y escandinavos ponían el dinero y dejaban que fueran gente local española, primero a comisión y después con participaciones en el negocio, los que llevaran hacia delante la construcción, promoción y venta de apartamentos, parcelas y casas.

Así, en la zona de la Axarquía surgieron los núcleos alemanes de Algarrobo Costa, Torrox Costa, Torre del Mar (si bien aquí, al tratarse también de uno de los lugares preferidos de los madrileños para las vacaciones estivales en apartamentos, la situación estaba más diluida). Igualmente, a nivel de chalés y de un público más potente, se crearon las urbanizaciones de Caleta del Sol, Rivera de Trayamar o La Sirena dónde hasta no hace mucho un alto porcentaje de los propietarios era de origen alemán, escandinavo y algo menos británicos, ya que estos se asentaban en Nerja o la Costa del Sol occidental. Los motivos que han traído a la Costa del Sol a la población alemana son principalmente tres: el clima (24\%), la calidad de vida $(20 \%)$ y la acogida de los españoles (12\%). El clima y la calidad de vidas son el gran exponente de estas poblaciones para asentarse en la Costa del Sol, especialmente porque son una población mayoritariamente jubilada que busca un mejor clima decisivo para su bienestar emocional y físico, así como las posibilidades de ocio que le brinda un mejor clima.

Durante muchos años veníamos a España de viaje y teníamos siempre el deseo de venir a vivir a España. Elegimos cerca de Málaga porque necesitábamos estar cerca de una gran ciudad, por la cultura. (...) nosotros también pensamos primero: "probamos un año" y tuvimos que alargar porque un año no era suficiente para valorar, y ahora tenemos un hogar que nos gusta por lo que aún seguimos aquí. (Participante nº2, mujer, 57 años).

5 La colonia de alemanes en este municipio es un gran aliciente para el resto de la población y además es un signo visible en la señalética de la ciudad (Pozo 2015). 
La vecina en nuestro alojamiento nos dijo que deberíamos ir a Andalucía, que Andalucía era muy bonita, y que deberíamos visitarla, y así acabamos hablando de si deberíamos comprar (una casa) allí o no comprar. Y al final nos decidimos a venir, a Málaga, a Benajarafe, y vimos la casita, la compramos y venimos todos los años de dos a tres meses aquí en Andalucía, de vacaciones (Participante no 4 , hombre, 75 años).

Decisiones que se materializan también en la adquisición de viviendas en la zona. Los datos desvelados en las encuestas indican que el $39 \%$ viven en casas de propiedad, un $42 \%$ en casas de alquiler y un $18 \%$ son propietarios de una segunda residencia. La interpretación de estos datos se debe a que el alquiler en la cultura alemana está bastante asentado, y el precio de la vivienda en Alemania es muy similar al de España: en algunos casos incluso más bajo. Lo que explica que el alemán siga prefiriendo alquilar y no estar atado a la hora de desplazarse por motivos profesionales o familiares y también de hacer vacaciones. Que un $42 \%$ viva en casas de alquiler tiene que ver también, o esencialmente, con el hecho de que el ciudadano alemán en su propio país prefiere utilizar este tipo de vivienda para mantener su libertad. Los datos de nuestras encuestas muestran que el $57 \%$ de los alemanes viven en casas de propiedad -para un 18\%, es una segunda residencia- y un $42 \%$ residen en casas de alquiler. Aunque pensemos que estos porcentajes están lejanos de la situación en España, donde el $84 \%^{6}$ tiene al menos una vivienda propia, o del $70 \%$ de la media europea, si los comparamos con la situación en Alemania, donde tradicionalmente se prefiere el alquiler y sólo un $45 \%{ }^{7}$ de los alemanes tiene vivienda propia, a pesar de que en los últimos años haya subido la tendencia a comprar más que a alquilar, podríamos entender que se están adaptando a las costumbres locales y mostrando su compromiso con el lugar donde viven.

El porcentaje de población activa por parte de los alemanes es relativamente bajo, el $21 \%$ son trabajadores con un empleo pagado y un $16 \%$ son autónomos, el resto son jubilados (59\%). Este bajo porcentaje de autónomos responde en gran medida, como ya se ha indicado, a que las posibilidades de trabajar en España para los alemanes son bastante limitadas, de ahí que o bien tengan empresas en torno al mercado inmobiliario y de construcción o en torno al idiomático de la traducción o bien ambos combinados.

Los planes de futuro de esta población a largo plazo en la Costa del Sol son claros: el 91\% señala que pretende quedarse a vivir aquí por ahora. Por tanto, se interpreta que la población alemana es ya parte del ecosistema cultural, mediático y económico de la Costa del Sol.

\subsection{La dieta mediática de los residentes alemanes en la Costa del Sol}

La población alemana en la Costa del Sol, según los resultados, se caracteriza por consumir los medios de comunicación de su país de origen $(87 \%$ y los medios de comunicación producidos para este grupo de residentes europeos en la Costa del Sol (87\%). Argumentan los encuestados que consumen ambas ofertas porque los medios de Alemania les dan una información general e informan del mundo entero (38\%) y de su país de origen (31\%), y los editados en la Costa del Sol

6 Véase para más información: https://www.elmundo.es/economia/vivienda/2017/05/22/5922af1b468aeb744e8b45a0.html

7 Véase para más información: https://www.faz.net/aktuell/finanzen/meine-finanzen/mieten-und-wohnen/nachrichten/warum-die-deutschen-eherzur-miete-wohnen-14743908.html 
les ofrecen información para su vida diaria (23\%) en nuestro país, les ayudan a conocer la Costa del Sol (18\%) y están en su idioma (17\%).

Este alto índice de consumo de sendos ecosistemas mediáticos puede deberse a que se complementan y sólo en su conjunto pueden satisfacer los intereses y necesidades informativas principales de los residentes alemanes. Según manifiesta el 65\% de los encuestados, los medios de su país de origen no les informan de la Costa del Sol. Y los medios de la Costa del Sol apenas les informan de lo que acontece en su país de origen o a nivel internacional. Y aclaran que consumen los medios editados en la Costa del Sol porque les ofrecen información para su vida diaria y les permiten conocer lo que acontece en el territorio en el que viven e informarse sobre las posibles actividades en la región y todas aquellas necesidades de ocio, cultura o prácticas en la vida cotidiana que desean o necesitan cubrir o realizar.

Los contenidos concretos que más les interesan son, por orden decreciente, las noticias sobre España (12\%), los eventos locales (11\%), las políticas locales (9\%); la cultura y tiempo libre (10\%). Así, los resultados de este apartado indican cómo una gran mayoría de los residentes alemanes muestran un interés por integrarse en la Costa del Sol y mantenerse al día de la comunidad a la que han decidido pertenecer.

Otros datos significativos son que frente al $76 \%$ de los encuestados que consideran que la información obtenida les sirve para conocer el sitio donde viven, un 55\% señala que la información aportada no les ayuda a resolver sus problemas del día a día como residentes extranjeros en la Costa del Sol. Así como, significativo es que la mayoría de los encuestados indican que consumen los medios producidos para la población objeto de estudio en la Costa del Sol porque están en su idioma, y un 59\% indica que estos medios no les ayuden a aprender español. Unos datos relevantes porque en las encuestas y los grupos de discusión los participantes han subrayado que no dominar el español es una de las principales barreras que imposibilitan su integración.

Los resultados de las encuestas evidencian que los medios más consumidos por los residentes alemanes son los impresos en papel. Actualmente se publican en la Costa del Sol 4 revistas y 3 periódicos íntegramente en alemán, junto a otros 6 medios plurilingües. Entre estos medios impresos los medios más consumidos por los encuestados son el Sur Deutsche Ausgabe y el Costa del Sol Nachrichten. Esta prensa tradicional quizás sea la más leída porque está disponible periódicamente en la mayoría de los puntos de venta de prensa con un repertorio extranjero, pero entendemos que los resultados también pueden estar influenciados por el hecho de que entre los encuestados existe un alto índice de jubilados (59\%) que seguramente serán más proclives a leer en papel que no on-line ${ }^{8}$.

Además hay que tener en cuenta que aunque estos dos medios sean de pago -a diferencia de la tendencia general en la prensa para extranjeros que es gratuita-, no dejan de ser los únicos medios de información "completa", en los que el fin publicitario es para el lector como mínimo secundario."[...] ahí está todo, eventos, anuncios, lo que pasa, contactos..." (Participante n5, mujer, 73 años), y ofrecen además una publicidad comercial seleccionada especialmente para las necesidades que suelen tener los alemanes residentes en la región, tanto en el sentido de su oferta como del de su atención al

8 SAZ-aktuell es un periódico exclusivamente digital. Spanien aktuell y Das aktuelle Spanienmagazin son más bien revistas con información cultural y de ocio y publicidad, la segunda sólo digital. Economía Hispano alemana / Deutsch-Spanische Wirtschaft es una igualmente una revista exclusivamente digital y de temática específica económica. 
potencial cliente en alemán o inglés. Los medios locales en español no representan generalmente una alternativa factible, ya sean audiovisuales o impresos, por la barrera lingüística, aunque no pocos alemanes se esfuerzan en consumirlos:

El locutor, como habla tan deprisa casi no le entendemos, al menos eso es lo que me pasa a mí. Pero se pueden leer las bandas inferiores y así uno puede hacerse una idea y tener más información que sólo con lo que uno oye (Participante, nº 7, hombre, 75 años).

El periódico Sur opta por una información en cierto modo "bilingüe" al incluir en su edición alemana de los jueves una edición especial del Sur en español, aunque no se trate realmente de una traducción de la versión alemana. A continuación, se reproduce una conversación procedente del grupo de discusión que profundiza en este aspecto:

Para nosotros son muy importantes, por las tardes, o en el médico... los hojeamos de principio a fin: cuando hay temporal, donde ha pasado algo... estas publicaciones son para nosotros muy importantes. Sobre todo para cuando pasan cosas, para contactos y también para eventos, y las cartas de los lectores, o abogados, respuestas... a veces, también sacar una receta, si no está mal. Para nosotros son interesante de principio a fin desde luego. (Participante nº 5, mujer, 75 años).

Son muy importante para saber lo que pasa aquí en el país. (Participante nº 4 , hombre, 75 años).

Yo también lo veo así, sobre todo para alguien que no hable español, están muy bien para poder conocer a groso modo un poco sobre política, lo que tu decías, lo que sucede... (Participante, $\mathrm{n}^{\circ}$ 1, hombre, 48 años).

También los artículos de viaje. (Participante no 5, mujer, 75 años).

Ah, sí, los artículos de viaje por ejemplo "Los Pueblos Andaluces”, esta (sección) me parece genial, descubrir estas pequeñas aldeas que casi nadie conoce, ni siquiera los españoles. Aparecen en una serie, cada semana un pueblo español diferente, o lo de Axarquía está muy bien eso (la información), simplemente por saber dónde se puede viajar. (Participante, $n^{\circ}$ 1, hombre, 48 años).

Es que si no, no nos enteramos de lo que pasa. (Participante no 5 , mujer, 75 años).

No sorprende que el nivel de satisfacción del grupo de discusión con estos medios no es tan alto como el que reportan las encuestas:

Imagen 4. Nivel de satisfacción con la prensa en alemán producida en la Costa del Sol

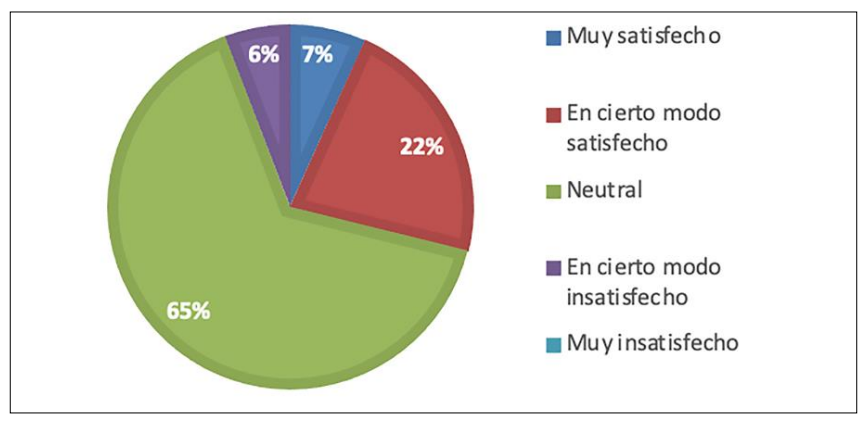

Fuente: elaboración propia 
El gran porcentaje de "neutrales" (65\%) se interpreta en parte debido a la gran cantidad de publicidad que tienen que incluir estos medios para poder sobrevivir. En el SUR alemán (Sur Deutsche Auusgabe), la mitad del contenido es informativo y la otra mitad es de publicidad cultural, de ocio y comercial. Resulta comprensible que por muy interesante que sea un periódico, el lector alemán no le dé una valoración muy positiva cuando la mitad de su contenido es publicidad, pero tampoco negativa, ya que parte de la publicidad es especialmente interesante para este colectivo como ya se indicaba. De hecho, sobre este aspecto concreto se les pregunta también en las encuestas y el $70 \%$ indica que la publicidad de estos medios les ofrece información útil.

Los comentarios del grupo de discusión confirman esta valoración neutral sobre la prensa más leída, pero también una postura más crítica con el resto de medios en alemán: "El 50\% está traducido y no tiene mucho contenido y tampoco está dirigido específicamente a los alemanes" (Participante ${ }^{\circ} 1$, Hombre, 48 años). La prensa local para alemanes se ajusta, por tanto, a las definiciones sobre periodismo de servicios entendida en el sentido que señala Diezhandino (1993) destinada esencialmente a ofrecer una información práctica, pero no fomenta de modo alguno la integración de estas personas en la sociedad española ni, en una interpretación más amplia, la cohesión europea.

Los comentarios personales en las encuestas confirman que para informarse sobre Europa y el proceso de construcción europea, o de fenómenos de máxima actualidad como el Brexit, los residentes alemanes no acuden a los medios publicados en la Costa del Sol en su idioma, sino que siguen acudiendo para esto a los medios de su país o, en el caso de los que dominen el español, a los medios españoles:

Con respecto a la información yo me informo con la prensa española, también la alemana, pero más la española, la alemana más en Internet. Varios medios. (Participante, n²2, mujer, 57 años).

Mi información la recojo tanto de la prensa española como de la alemana, y es muy interesante, porque los puntos de vista y las perspectivas son muy diferentes. (Participante $n^{\circ} 1$, hombre, 48 años).

Nosotros lo hacemos exactamente igual a pesar de que nuestro español no es muy bueno, pero vemos directamente las noticias en televisión. Y de la mitad más o menos si nos enteramos. Y la perspectiva u opinión no se forma solamente de las noticias: en primer lugar, nosotros tenemos una visión muy diferente, como ya hemos comentado, y también tiene que ver con nuestro círculo de amigos también es así, los vemos a menudo, hablamos, discurrimos, la política es un tema recurrente. (Participante $n^{\circ} 6$, mujer, 60 años).

Esta función primordial de los medios extranjeros de la Costa del Sol como periodismo de servicios coincide con la del estudio sobre los medios extranjeros destinados a los alemanes en Canarias realizado por Dorn Padilla (2012).

En el grupo de discusión también se ha puesto de manifiesto las lagunas que presentan para estas poblaciones la información relativa a la Unión Europea publicada en los medios de su país de origen y españoles: indican que no se orienta a la problemática de índole más práctica de los ciudadanos europeos, especialmente apuntando que no encuentran en el discurso soluciones a sus problemas del día a día. Un déficit que argumentan en un ámbito que es fundamental para el sentimiento de integración como es el de las elecciones municipales, en las que todo ciudadano alemán puede participar. Quizás por este motivo, en los datos obtenidos en la encuesta, del 85\% de alemanes registrados en su ayuntamiento, sólo un $34 \%$ ha votado alguna vez en las elecciones locales españolas. 
Otra de las carencias o críticas que exponen sobre el tratamiento que se hace de la información sobre Europa en la prensa en general, es que los temas o la agenda mediática están demasiado centradas en determinados temas de actualidad, como el Brexit o la inmigración.

Mira lo que pasa en Alemania, no hacen más que hablar de los emigrantes, y los idiotas no son capaces de hablar de los problemas de verdad, como, por ejemplo, la falta de pisos, no hay suficientes viviendas en Alemania, es una locura. La gente no puede conducir con sus coches porque la política corrupta, la industria automovilística, sobre ese tema no habla nadie, cuando hay problemas se habla de los emigrantes y no de los verdaderos problemas. Que los servicios se están descuidando...tenemos problemas de verdad en la sociedad y están hablando de lo que no es un problema. (Participante nº 3, hombre, 57 años).

Cierto, la (crisis) de las guarderías, los colegios. (Participante $n^{\circ} 7$, mujer, 75 años).

\subsection{El proceso de integración de los migrantes alemanes en el actual contexto europeo}

Tanto el grupo de los residentes alemanes jubilados como el de los profesionalmente activos han tenido desde un inicio una postura claramente europeísta y comunitaria y han estado fielmente comprometidos con el proyecto europeo. Para el 90\% de los encuestados es muy importante ser ciudadano de la Unión Europea. Esto lo confirma de manera aún más contundente el grupo de discusión. La fortaleza de sus comentarios no deja duda sobre la capital importancia que tiene para esta población pertenecer a la Unión Europea. El proyecto europeo les facilita decididamente su establecimiento en la Costa del Sol, tanto desde el punto de vista burocrático como cotidiano, les motiva a integrarse y favorece la cohesión:

Pienso que nosotros hemos tenido una experiencia muy positiva dentro de la Unión Europea. Hemos vivido en una ciudad fronteriza, en Aquisgrán, y está justo en la frontera, con Bélgica y Holanda como vecinos. Y recordamos los tiempos en los que aún había fronteras y comparando cómo es ahora, para nosotros es algo muy positivo. Para mí al menos, el hecho de que nosotros ahora vivamos en España, no hubiésemos podido gestionarlo si no existiera la UE, de la forma en la que está estructurada. (Participante, nº2, mujer alemana 57 años).

Nosotros nos sentimos tan intensamente europeos, que hablo por nosotros al menos cuando digo que no podemos entender que Cataluña se quiera separar, o los ingleses, no lo puedo entender porque esto proporciona tantas ventajas... (Participante, n6, mujer alemana, 60 años).

O los ingleses. (Participante, $\mathrm{n}^{\circ} 1$, hombre alemán, 48 años).

$\mathrm{Si}$, o los ingleses. Estoy totalmente de acuerdo..., simplemente cuando uno lo compara con antes... ahora uno va a cualquier sitio, la moneda es la misma, uno puede comparar fácilmente, los precios, los servicios ... (Participante, nº2, mujer alemana 57 años).

Yo creo que todo aquel que no lo ha entendido, es porque pretende dar marcha atrás 50 años. Está ya todo tan entrelazado, todo, se ve por ejemplo en las páginas oficiales de los británicos: informan de que ahora hay 30.000 leyes que hay que reescribir o volver a votar. Eso no es posible, no es algo que pueda pasar. Y además no es algo que sea sólo un tema práctico, de la vida en el día a día tiene que ver también con la política a un nivel más alto. Con respecto a nosotros, a nuestra edad, ya no es algo comprensible vivir sin ello. (Participante, nº3, hombre, 57 años). 
Yo tampoco tengo claro que ahora viviría aquí sin la UE. Llegué a España con 100 Marcos (alemanes) en el bolsillo, por aquel entonces eran marcos, tuve estrés durante dos semanas con las autoridades, y me hice autónomo. En España. Y podía empezar a trabajar. (Participante, $n^{\circ} 1$, hombre alemán, 48 años).

Eso no hubiese sido posible de otra manera. (Participante, n5, mujer, 73 años).

Es probable que hubiese sido posible, pero no sé, creo que no hubiese aceptado ese reto, dadas las circunstancias, porque de esta forma te lo ponen en bandeja [sic]. Y cuando me jubile solo tengo que ir a Alemania, aportar los documentos de aquí que demuestren que he trabajado, y ellos mismos calculan y complementan lo que yo he trabajado y recibiré una pensión europea ¿hay algo mejor? Creo que no. Mi información la recojo tanto de la prensa española como de la alemana, y es muy interesante, porque los puntos de vista y las perspectivas son muy diferentes. (Participante, nº1, hombre alemán, 48 años).

Los datos obtenidos revelan cómo el trasfondo histórico personal junto a una visión muy positiva acerca de Europa es y ha sido un factor clave para fijar su residencia en España, pero al mismo tiempo, que el proceso de integración difícilmente puede llegar a ser del todo pleno. Los resultados muestran que el 59\% de los encuestados se siente siempre "como en casa" en la Costa del Sol, un 35\%, "la mayoría del tiempo", y sólo un 5\%, "a veces", y "nunca", el 1\%. La percepción que tienen los alemanes sobre la integración del resto de residentes europeos es bastante distinta: un $60 \%$ se siente "en cierto modo integrados" y un 35\% "poco integrados".

$\mathrm{Al}$ analizar la actitud de los alemanes por integrarse y los factores que aumentan o frenan su proceso de integración en la Costa del Sol, las encuestas indican que el $62 \%$ ha ido alguna vez a clases de español, el $90 \%$ tiene amigos o conocidos españoles y que el $59 \%$ pertenece a un club deportivo, cultural o social. Estas asociaciones suelen tener un papel importante en el proceso de integración de los conciudadanos, en el caso de nuestros encuestados alemanes, sin embargo, hay que relativizar bastante estos resultados porque si comprobamos la situación detrás de los datos de la encuesta, constataremos que las asociaciones a las que pertenecen están formadas esencialmente por compatriotas y algún que otro europeo. Algo similar sucede al analizar detenidamente el dato de que un $90 \%$ tiene amigos o conocidos españoles, ya que la mayoría se quejan de la dificultad para mantener una relación más estrecha o continua con estos, por lo que un $51 \%$ mantiene sus relaciones sociales cotidianas con una mezcla de personas de su país de origen, españoles y ciudadanos de otros países; un 25\% las mantiene sólo con sus compatriotas y un $4 \%$ exclusivamente con residentes de otros países.

Un elemento que favorece la integración en la sociedad española y las relaciones con las otras nacionalidades lo constituyen los medios extranjeros producidos en la Costa del Sol. Un 65\% de los encuestados alemanes cree que estos les ayudan en su proceso de integración a la sociedad española, y un $68 \%$, que igualmente fomentan la unidad entre los miembros de las diferentes comunidades extranjeras residentes en la región. 
Imagen 5. Opinión de los encuestados sobre si creen que los medios para extranjeros fomentan la integración entre la comunidad extranjera y la sociedad española

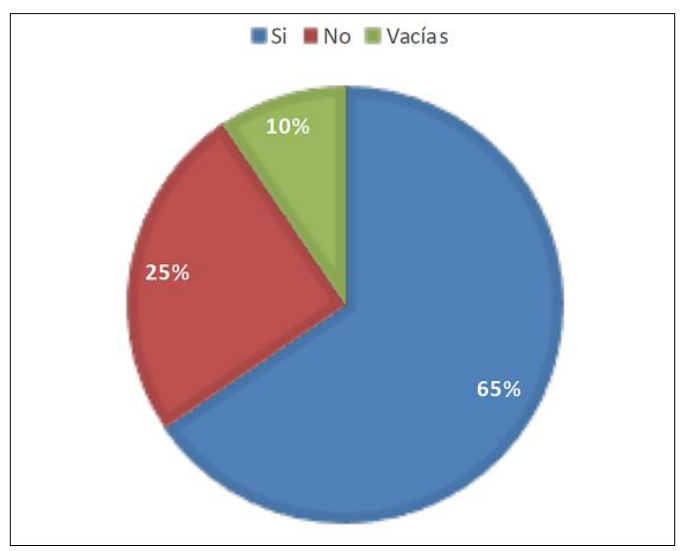

Fuente: elaboración propia

Imagen 6. Opinión de los encuestados sobre si creen que los medios para extranjeros fomentan la integración entre los miembros de comunidades extranjeras que viven en la Costa del Sol

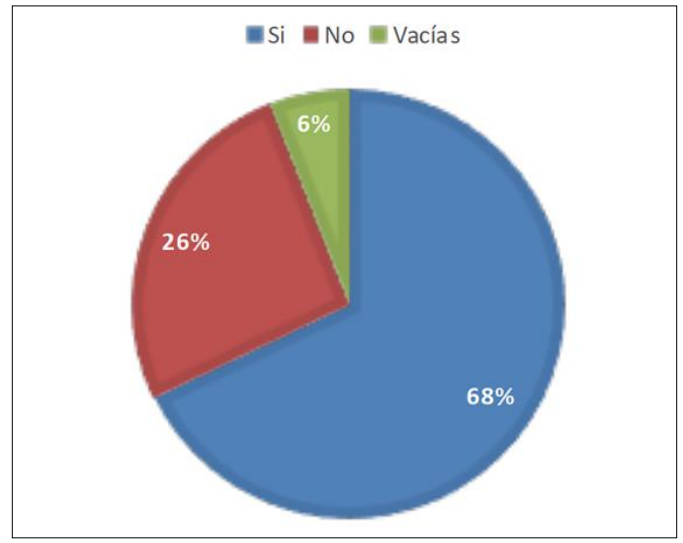

Fuente: elaboración propia

Desde el grupo de discusión se obtienen algunas claves que explican qué factores aumentan o dificultad su relación con los españoles. Como factores positivos destacan, por ejemplo, vivir en urbanizaciones de vecinos con mayoría de españoles o tener una relación sentimental con una pareja española (lo que posibilita el contacto con la familia y aumenta la red de contactos). De esta forma, aquellos participantes casados con parejas españolas apuntan que su círculo social "son un 99\% español" (participante, no 1 , hombre, 48 ), pero para aquellos que su pareja es alemana es todo lo contrario. "Es muy 
difícil, yo también trabajo desde casa y es muy difícil entrar en contacto con españoles, hay algunos españoles con los que tengo contacto, pero los demás son alemanes o ingleses" (Participante, n²2, mujer, 57 años).

Otro aspecto de la escasa integración se debe a ciertos aspectos culturales o costumbres de los españoles, por ejemplo, la conexión familiar que hace difícil que pasen tiempo con otras personas más allá de su familia.

Creo que aquí la vida con la familia es una prioridad, muy importante para los andaluces, esto no es algo español, creo que es algo andaluz, y es muy difícil porque la gente no tiene tiempo para estar con nosotros. Los andaluces pasan todo el tiempo libre con su familia y de esta forma no tienen prácticamente tiempo libre para pasarlo con amigos u otras personas: no solo extranjeros, pueden ser otros españoles.; no hay sitio para nuevos contactos (Participante, $\mathrm{n}^{\circ} 1$, hombre, 57 años).

Otro factor obstaculizador de las relaciones que comenta el grupo de discusión es la escasa costumbre que existe en España de invitar a las amistades a su casa, ni siquiera a quienes previamente les han invitado a la suya. Los asistentes destacan que la cultura española, al contrario de la alemana es más proclive a organizar los encuentros y celebraciones fuera del domicilio familiar:

Pero es muy peculiar con los españoles, a nosotros nos gusta invitar a amigos a casa y hacemos una barbacoa en el jardín o algo así, tenemos contacto con españoles, como acabas de decir tú, son nuestros vecinos más cercanos, pero nunca nos invitarían más allá de su terraza. (Participante, nº7, hombre, 75 años).

Esa es la diferencia, es decisivo. (Participante, $\mathrm{n}^{\circ} 2$, mujer, 57 años).

Lo mismo tiene que ver con que los edificios son muy pequeños, y es difícil tener invitados, los españoles. (Participante, $\mathrm{n}^{\circ} 7$, hombre, 75 años).

Pero eso está arraigado en su cultura. (Participante, nº2, mujer, 57 años).

La familia está en primer lugar, y salen con su familia los fines de semana y no queda más tiempo para iniciar otros contactos. El único que habla español con nosotros es el administrativo (de la comunidad). (Participante, no7, hombre, 75 años).

El asentamiento de la población alemana en la Costa del Sol, debido o a pesar a todo esto, no siempre es un éxito y no pocos alemanes vuelven a su país tras vivir o intentar sobrevivir aquí. Generalmente los retornos emocionalmente involuntarios no están motivados por cuestiones sociales sino laborales o de edad. Los alemanes que intentan asentarse trabajando en España constatan pronto que las condiciones de trabajo y las remuneraciones son bastante peores que en Alemania:

Los sueldos no están igualados, y las condiciones no son fantásticas, no tienes la misma seguridad como en Alemania, aquí en comparación con Alemania hay pocos derechos laborales, y cuanto más joven eres, o si eres muy joven, está bien, puedes estar unos años, pero a partir de los veinti-tantos o los veinti-muchos, incluso, cuando piensan en el futuro, ya no es posible. (Participante, n³, hombre, 57 años).

Los planes a largo plazo de los residentes alemanes en la Costa del Sol son claros: el 91\% señala que pretende quedarse a vivir aquí por ahora, por lo que los dirigentes de los medios de comunicación y las instituciones europeas deberán seguir considerándolos parte del ecosistema cultural, mediático y económico de la Costa del Sol y prestarles en el futuro una mayor atención. 


\section{Conclusiones}

Los objetivos de esta investigación se han centrado en el papel que desempeñan los medios extranjeros para la población alemana en la Costa del Sol, en su proceso de integración europea en esta área geográfica, y en identificar el perfil de las características socio demográficas de estas audiencias. Una investigación que forma parte del proyecto I+D+i "Medios de comunicación y construcción europea: estudio sociocomunicativo de los residentes (comunitarios en el sur de España y de Portugal (CSO2015-65837-R)".

La principal conclusión obtenida de los datos recopilados a través de encuestas y de un grupo de discusión a la población alemana, residente en la Costa del Sol, es que estos medios ayudan y son necesarios en su proceso de integración en esta área geográfica. Si bien, podrían aumentar esta función si atendiesen a las demandas informativas de estas audiencias y a los principales problemas que obstaculizan su integración.

La función integradora la cumplen parcialmente porque realizan un periodismo de servicios. Y es que informan de aspectos prácticos sobre: eventos culturales, turísticos y festivos de la región, dirigidos a la población autóctona pero de gran interés también para los turistas y residentes extranjeros que busquen el entretenimiento; cómo contactar con los residentes autóctonos o con sus compatriotas en función de sus intereses; o cómo localizar servicios específicos para los residentes extranjeros. Esta información la consideran útil y muy necesaria porque es lo único que tienen en su idioma para estar al día de lo que acontece en la Costa del Sol y porque les ayudan a integrarse en la sociedad española y extranjera. No obstante, demandan que se informe sobre asuntos que les ayuden a resolver sus problemas diarios como residentes de la Unión Europea en la Costa del Sol, identificando esta como una de las principales deficiencias de los medios extranjeros. Deficiencia que se podría subsanar prestando más cobertura a aquellos acontecimientos en los que esta audiencia pueda participar como residentes (por ejemplo, en las elecciones municipales) o hacer aún más hincapié en la información que tiene que ver con pautas y trámites a su condición de residentes europeos.

La audiencia alemana tanto jubilada como la profesionalmente activa de una y otra zona de la Costa del Sol muestra un gran espíritu europeísta y valoran las ventajas del proyecto europeo para integrarse en otras comunidades, porque las están experimentando día a día. Hecho por el que está altamente interesada en las cuestiones o acontecimientos europeos y del resto del mundo. Sin embargo, los medios extranjeros no cubren esta demanda, motivo por el que se acercan a otros dos ecosistemas mediáticos: la prensa de su país de origen y los medios españoles. Si bien, también destacan que estos dos ecosistemas presentan deficiencias. La principal, que abordan temas generales y tampoco se centran en asuntos que interesan a los residentes europeos.

Los medios españoles sólo son consumidos por quienes han aprendido español, siendo el desconocimiento de esta lengua su principal barrera a la integración. Aunque otros factores que afectan a la integración son vivir en comunidades conformada principalmente por alemanes, tener una pareja de la misma nacionalidad, tener un trabajo que sólo le permita relacionarse con alemanes u otras nacionalidades extranjeras, o algunas costumbres de la población española. Ayudar a superar estos problemas desde un medio de comunicación resulta limitado, pero se deben pensar propuestas que ayuden a mejorar la integración o contacto con la sociedad española. Es así, que la hipótesis de partida se ha cumplido. 
En este contexto, se considera que quizás estos medios deban configurarse como la punta de lanza de estos colectivos en su integración, ahora que se disponen de datos que ayudan a esto, al tiempo que pueden mejorar su negocio. Y es que la configuración actual de estos medios, como indica Rosell (2008), es que los directivos de estas empresas trabajan con la idea de que el futuro de los "medios étnicos" está condicionado por el propio colectivo, en consecuencia, limitado al tiempo que dure su proceso de integración hasta que no haya diferencias con el resto de los españoles. Es decir, que estos medios más que responder a las necesidades y requerimientos de su audiencia parecen ir a la estela de esta ciudadanía. No obstante, aumentar la integración a través de los medios extranjeros, cuando tomen conciencia de su importante labor como hilo conductor para la integración de la población alemana en la Costa del Sol quizás, sea sólo cuestión de tiempo. Observamos en este estudio la importancia de escuchar a la audiencia, y en el caso de los alemanes que residen en la Costa del Sol, demuestran que son una población que está firmemente convencida de seguir viviendo en esta zona, por tanto, se refuerza nuestra propuesta de que los medios de comunicación para extranjeros deben introducir importantes cambios para atender a las necesidades de estas poblaciones y mejorar su proceso de integración puesto que la Costa del Sol se ha convertido, a fin de cuentas, en su hogar.

Finalmente nos gustaría apuntar hacia futuras investigaciones que se derivan de este trabajo. Estas líneas analizarán el proceso de construcción del mensaje periodístico y las temáticas ofrecidas en la prensa extranjera objeto de estudio de esta investigación a través de la entrevista y análisis de los directores de los medios de comunicación analizados con el fin de paliar las deficiencias y optimizar las demandas de integración europea de la población residente alemana en la Costa del Sol.

\section{Financiación}

Este artículo ha sido financiado dentro del proyecto "Medios de Comunicación y construcción europea: estudio sociocomunicativo de los residentes comunitarios en el sur de España y de Portugal (CSO2015-65837-R)” financiado por el Ministerio de Ciencia, Innovación y Universidades.

\section{Referencias bibliográficas}

Acirón, R. (1997). Canarias: prensa y turismo. El turismo como fenómeno de comunicación. Tenerife: Ediciones Idea. Betty, C. (1997). Language problems of older British migrants on the Costa del Sol. Generations Review 7, pp. 10-11.

Betty, C. y Durán, R. (2009). "Retired British expatriates with reference to the importance of social clubs and integration in local Spanish society. A case study Benalmádena”. Observatorio Europeo de Gerontomigraciones.

Blau, J. (1998). “Immigrant Communities and Their Newspapers in America, 1850-1930." Sociological Analysis 1, pp. 13-24. Brückner, G. (2008). "Glocalización comunicativa y prensa local extranjera en España: La visión de los residentes alemanes de la Costa Blanca." En Relaciones hispano-alemanas: prejuicios y estereotipos, encuentros y desencuentros: un balance. Alicante: Universidad de Alicante, pp. 115-123. 
Delgado Peña, J. J. y Sortino Barrionuevo, J. F. (2018). Evolución y desarrollo del turismo residencial británico en la provincia de Málaga y Costa del Sol (Málaga, España). En Mazón, T. (ed.). Turismo residencial. Nuevos estilos de vida: de turistas a residentes. Universitat D’Alacant, pp. 187- 198.

Diezhandino, M.P. (1993). “El 'periodismo de servicios', la utilidad en el discurso periodístico”. Análisi 15, pp. 117-125.

Dorn Padilla, M. T. (2012). “Prensa y turismo alemán en Canarias. Comunicación”. En Actas del IV Congreso Internacional Latina de Comunicación Social. Tenerife: Sociedad Latina de Comunicación Social. Disponible en: http://www. revistalatinacs.org/12SLCS/2012_actas/042_Dorn.pdf

Fernández, A. y Mendoza, A. (2007). "La actividad turística en el territorio andaluz. Establecimiento de indicadores, distribución y evolución de los mismos.” Boletín de la A.G.E. 44, pp. 117-146.

García Galindo, J. A. (1998). "El papel de los medios de comunicación en la configuración y promoción de la Costa del Sol”. Historia de La Costa del Sol. Málaga: Diario Sur, pp. 233-240.

García Galindo, J. A., y López Romero, L. (2013). "La prensa en lengua extranjera en Andalucía: estado de la cuestión”. Jornadas Premières rencontres internationales du réseau Transfopress, 28-29 de noviembre, París.

García Galindo, J. A; López Romero, L. (2018). Los medios de comunicación en lengua extranjera. Editorial Comares.

López Romero L. y Serrano Porras, J. (2016). "Los orígenes de la prensa en lengua extranjera en Málaga”. Historia y Comunicación social, vol. 21 (1) pp. 175-188. http://dx.doi.org/10.5209/rev_HICS.2016.v21.n1.52690

López Romero, L. (2009). Prensa y comunidad extranjera en España. Estudio descriptivo y analítico del caso malagueño. Málaga: Quiasmo.

Gómez Mompart, J. L. (2008). "Diversidad latina y comunicación panhispana en la prensa en español de Nueva York al inicio del siglo XXI”. Doxa Comunicación 6, pp.15-51. DOI: 10.31921/doxacom

Gómez-Escalonilla, G. y Santín, M. (2009). "Voces latinas: el periodismo de la inmigración”. En actas del I Congreso Internacional Latina de Comunicación Social. Tenerife: Sociedad Latina de Comunicación Social, pp. 1-11.

--González Cortés, M. E. (2009). "La consolidación de las publicaciones para extranjeros en España", en RLCS, Revista Latina de Comunicación Social, 64, pp. 708-724. La Laguna (Tenerife). Universidad de La Laguna. Disponible en: http://www.revistalatinacs.org/09/art/856_UMA/57_91_Maria_Eugenia_Gonzalez_Cortes.htm. DOI: 10.4185/ RLCS-64-2009-856-708-724

González Cruz, I. (1991). “The Canary Islands review: el periódico inglés de Las Palmas”. Parabiblos: cuadernos de biblioteconomía y documentación 5-6, pp. 39-49.

Lacroix, J. M. (1998). Anatomie de la presse ethnique au Canada. Burdeos: Presses Universitaires de Bordeaux.

Lakssfoss, A. E (2018). De turistas a residentes. Los inmigrantes no laborales. En Mazón, T. (ed.). Turismo residencial. Nuevos estilos de vida: de turistas a residentes. Universitat D`Alacant, pp. 45-50.

Marimon Riutort, A. y Vicens, C. (2013). "Entre la culture et le tourisme, la presse en langues étrangeres dans les îles Baléares". Jornadas Premières rencontres internationales du réseau Transfopress, 28-29 de noviembre, París. 
Martín Hernández, U. (1990). "La presencia extranjera en el escenario periodístico isleño (en torno a 1900)". Tebeto: anuario del Archivo Histórico Insular de Fuenteventura (Islas Canarias) 3, pp. 135-146.

Mas Giralt, R. (2017). "Onward migration as a coping strategy? Latin Americans moving from Spain to the UK post-2008”. Population, Space and Place, 23(3). DOI:10.1002/psp.2017.

Maza, A., Villaverde, J. y Hierro, M. (2013). “Explaining the settlement patterns of foreigners in Spain”. Applied Geography, 40(juin), pp. 11-20. DOI: 10.1016/j.apgeog.2013.01.004.

Mazón, T. (2018). Turismo residencial. Nuevos estilos de vida: de turistas a residentes. Universitat D’Alacant.

Millares, J. (2018). "De turistas a residentes. Relaciones y percepciones de los extranjeros europeos en las áreas rurales del interior de Mallorca de 1996 a 2008”. En Mazón, T. (ed.). Turismo residencial. Nuevos estilos de vida: de turistas a residentes. Universitat D’Alacant, pp. 187-198.

Ocaña, M.C. y Larrubia, R. (2012). “Residentes extranjeros y crecimiento demográfico en el territorio andaluz." Boletín de la A.G.E. 60, pp. 45-76.

Oliveau, S., et al. (2019). "Mapping foreign nationals in Spain: an exploratory approach at local level". Genus, vol. 75, no 1, p. 5. DOI: $10.1186 / \mathrm{s} 41118-018-0047-5$

O'Reilly, K., (2009). "Hosts and guests, guests and hosts: British residential tourism in the Costa del Sol”. Cultures of Mass Tourism: Doing the Mediterranean in the Age of Banal Mobilities. Farnham: Ashgate, pp. 129-142.

Penalva, C. y Brückner, G. (2008). "Comunicación intercultural. Un estudio de caso sobre la prensa local extranjera en España.” Revista Internacional de Sociología 50, pp. 187-211. https://doi.org/10.3989/ris.2008.i50.101

Pozo, J. C. (2015). “Torrox la pequeña Alemanía de Málaga”. Andalucía Lab. Centro de Innovación Turística. Disponible en: https://www.andalucialab.org/blog/torrox-comunidad-de-alemanes/

Retis, J. (2008). “Espacios mediáticos de las migraciones en Madrid: Génesis y Evolución”. Observatorio de las Migraciones y la Convivencia Intercultural de la ciudad de Madrid. Madrid.

Rodriguez, V., Fernandez-Mayoralas, G., y Rojo, F. (2004). “International retirement migration: retired Europeans living on the Costa del Sol, Spain”. Population Review, 43(1), pp. 1-36. DOI:10.1353/prv.2004.0009.

Rosell, M.M. (2008). “La prensa para inmigrantes se profesionaliza” Cuadernos de Periodistas 13, pp. 81-89.

Sáiz Díaz, M. J. (2016). "Preferencias televisivas de entretenimiento de los jóvenes británicos residentes en la Costa del Sol” Actas del I Congreso Internacional Comunicación y Pensamiento. Comunicracia y desarrollo social. Sevilla: Egregius, pp. 714-729.

Santos Díez, M. T. y Pérez Dasilva, J. (2012). "Prensa gratuita para inmigrantes en el País Vasco: una lucha por la supervivencia”. Doxa Comunicación: revista interdisciplinar de estudios de comunicación y ciencias sociales, ${ }^{\circ} 14$, pp. 5973. Disponible en: http://www.doxacomunicacion.es/es/hemeroteca/articulos?id=127 DOI: 10.31921/doxacom 
Santos Díez. M. T. (2008). "Periódicos gratuitos para inmigrantes. Noticias de acá y de allá." Estudios sobre el mensaje periodístico 14, pp. 605-616. Taillefer de Haya, L. (2005). “La edición en inglés de un periódico español: SUR in English”. La traducción periodística. Cuenca: Ediciones de la Universidad de Castilla-La Mancha, pp. 259-287.

Woube, A. (2017). "Living with Change Among a Transient Population: Narratives and Practices of Collective Belonging among Swedish Migrants on the Costa del Sol in Spain”. Nordic Journal of Migration Research, special issue article, pp. 148-155. DOI: 10.1515/njmr-2017-0024 
